\title{
Preparative Isolation and Purification of Altertoxin I from an Alternaria sp. by HSCCC
}

\author{
Dejun $\mathrm{Hu}^{1}$, Miao Liu ${ }^{2}$, Xing Xia ${ }^{3}$, Daijie Chen ${ }^{4}$, Fengsheng Zhao ${ }^{1}$, Mei Ge ${ }^{4, 凶}$ \\ 1 Department of Pharmacy, Shanghai Jiao Tong University, 200240 Shanghai, China \\ ${ }^{2}$ School of Life science and Biopharmacy, Shenyang Pharmcuetical University, 110016 Shenyang, China \\ ${ }^{3}$ Shanghai Institute of Pharmaceutical Industry, 200040 Shanghai, China \\ ${ }^{4}$ Shanghai Health Creation Center of Biopharmaceutical R\&D, 201203 Shanghai, China; E-Mail: hocbred@gmail.com
}

\begin{abstract}
Altertoxin I (ATX I) is one of the common mycotoxins produced by genus Alternaria which is a common food pathogen of fruits and grains. To prepare enough quantity of pure ATX I for further research of mutagenicity and toxicology tests, a novel method using preparative highspeed counter-current chromatography (HSCCC) was developed. The ethyl acetate crude extracts of the acetone washes obtained after fermentation of Alternaria sp. was separated using a two-phase solvent system composed of $n$-hexane-ethyl acetate-methanol-water $(2: 5: 5: 6, v / v)$. Collected fractions were analyzed by $L C$ and identified by El-MS and NMR analysis. The technique can isolate mg levels of the target compound per run.
\end{abstract}

\section{Keywords}

High-speed counter-current chromatography (HSCCC)

Mutagenicity

Altertoxin I

Alternaria sp

\section{Introduction}

Altertoxin I, also known as ATX I, is a mycotoxin produced by molds of the genus Alternaria. Alternaria is a common food pathogen responsible for the spoilage of fruits, vegetables, grains and nuts [1-5]. A variety of toxins produced by Alternaria have been associated with mutagenicity of Ames Salmonella and adverse health effects, including esophageal cancer in Linxian County, China as well as in areas of Southern Africa [6-10]. Numerous cases of human toxicity, basically due to the ingestion of food contaminated by species of the Alternaria can be found described in the bibliography [11]. Alternaria causes serious damage to fruits and produces ATX I, a common mytotoxin with low levels of contamination that have been detected in a diverse variety of foodstuffs which range from wheat to fruit juices [3, 12-16]. Among the major Alternaria toxins, ATX I is the most active mutagen in the Ames Salmonella typhimurium assay, and exhibits acute toxicity in mice and cytotoxicity to bacterial and mammalian cells $[6,9,17-20]$. ATX I as the major mutagenic species could pose a potent carcinogenic health hazard when combined with a diet high in nitrites and nitrates [6,9]. Contamination of fruits and grains with ATX I will create a food safety risk. Studies on mutagenicity, toxicology and detection of ATX I are, therefore, a priority for the food/feed industry and regulatory agencies. The study often requires relatively large quantities of pure ATX I.

Isolation of ATX I was done by a multi step protocol based on repeated column chromatography, often followed by a final purification on preparative 
thin layer chromatography (TLC) $[17,21,22]$. Currently, there is no practical method for ATX I synthesis, and the commercially available standard is costly. In general, methods currently available for purification of ATX I are time-consuming, require multiple steps, and often generate large amounts of organic solvent wastage.

High-speed counter-current chromatography (HSCCC) is a liquid-liquid partition chromatography process developed by Ito $[23,24]$. The technique has been widely used in preparative separation of natural products with minimum sample preparation and cleanup procedures $[25,26]$. Its theory is well understood and solvents mixtures are known for almost any analyte polarity [27]. The separation and purification of ATX I from extract of the Alternaria culture by HSCCC has not been reported so far. The objective of this study was, therefore, to develop a novel method using HSCCC for more efficient purification and recovery of ATX I from the Alternaria culture.

\section{Experimental}

\section{Apparatus}

The HSCCC instrument employed in this study was a TBE-300A high-speed counter-current chromatograph (Tauto Biotechnique, Shanghai, China) with three PTFE (Polytetrafluoroethylene) multi-layer coil separation column connected in series (I.D. of the tubing = $1.8 \mathrm{~mm}$, total volume $=300 \mathrm{~mL}$ ) and a $25 \mathrm{~mL}$ sample loop. The revolution radius was $5 \mathrm{~cm}$, and the $\beta$ of the multilayer coil varied from 0.6 at internal terminal to 0.8 at the external terminal ( $\beta=r / R$ where $\mathrm{r}$ is the distant from the coil to the holder shaft, and $\mathrm{R}$ is the revolution radius or the distant between the holder axis and central axis of the centrifuge). The revolution speed of the apparatus can be regulated with a speed controller in the range between 0 and 1,000 rpm. An HX 1050 constant-temperature circulating implement (Beijing Boyikang Lab Instrument, Beijing, China) was used to control the separa- tion temperature. The effluent was continuously monitored with a UV-Monitor 8823B (Beijing Binta Instrument Technology Co., Beijing, China) at $254 \mathrm{~nm}$. The data were collected with a model N2010 chromatography workstation (Zhejiang University, Hangzhou, China). The fractions were collected in intervals of $4 \mathrm{~min}$ with a DC- 1000 Model auto-fractional collector (Eyela, Tokyo, Japan).

Agilent 1100 LC system was used, which included a G1311A QuatPump, a G1315B UV-vis photodiode array detector, a G1313A Auto-sampler, a G1332A degasser and Agilent LC workstation.

\section{Reagents, Cultural Media and Alternaria sp. Isolate}

All organic solvents used for preparation of the crude extract and HSCCC separation were of analytical grade (Shanghai Chemical Reagent Corporation, Shanghai, China). Acetonitrile used for LC was of chromatographic grade (Merck), and distilled water. DMSO was used as the solvent for NMR determination. Potato dextrose agar (PDA), peptone and starch were purchased from Beijing Comwin Pharm-Culture Corporation (Beijing, China). ATX I and Alternaria sp. CPCC 480171 were obtained from Shanghai Health Creation Center of Biopharmaceutical R\&D (Shanghai, China). The fungus was stored on PDA slant at $4{ }^{\circ} \mathrm{C}$, and grown on PDA plates at $27^{\circ} \mathrm{C}$ for 5 days before.

\section{Fermentation of Alternaria sp. and Extract Preparation}

Alternaria sp., CPCC 480171 was cultured in $500 \mathrm{~mL}$ flasks containing potato-dextrose liquid medium (potato $200 \mathrm{~g}$, dextrose $20 \mathrm{~g}, \mathrm{H}_{2} \mathrm{O} \quad 1,000 \mathrm{~mL}$, total volume $200 \mathrm{~mL}$ ) for 4 days at $25^{\circ} \mathrm{C}$, $120 \mathrm{rpm} .800 \mathrm{~mL}$ of the resultant culture was used to inoculate a 30-L GUJS-30C fermenter (Zhenjiang Oriental Biotech Instrument Co, China) containing $18 \mathrm{~L}$ of SDP medium (peptone 2\%, dextrose $1 \%$ and starch $2 \%, \mathrm{pH} 6.5$ ) plus $0.1 \%$ corn oil. After the sterilization cycle at $121{ }^{\circ} \mathrm{C}$ for $45 \mathrm{~min}$, the medium was cooled to $27^{\circ} \mathrm{C}$ and inoculated. The fermenter was incubated at $27{ }^{\circ} \mathrm{C}$ and maintained at 0.5 bar overpressure with an agitator speed of $300 \mathrm{rpm}$ and an air flow rate of $10 \mathrm{~L}$ air $\mathrm{min}^{-1}$.

After 5 days the cultured broth was harvested and centrifuged. The biomass of the fungal strain was collected and extracted with acetone $(3 \mathrm{~L} \times 3)$ to yield a crude extract that was a dark reddish solid. The dried extract was dissolved in $1.5 \mathrm{~L}$ water and then re-extracted with ethyl acetate for three times. $24.7 \mathrm{~g}$ ethyl acetate extract was obtained after evaporated to dryness under vacuum at $40{ }^{\circ} \mathrm{C}$ with a rotary evaporator (N-1000, Eyela, Japan). The ethyl acetate crude extract was stored at $4{ }^{\circ} \mathrm{C}$ before HSCCC separation.

\section{Selection of Two-Phase Solvent System}

The two-phase solvent system was selected according to the partition coefficient $(K)$ value of the target compound. The $K$ values of ATX I were determined by LC as follow: $1 \mathrm{mg}$ of the crude extract was added to a test tube, to which $2 \mathrm{~mL}$ of each phase of a preequilibrated two-phase solvent system was added. The test tube was then capped and shaken vigorously for $1 \mathrm{~min}$ to thoroughly equilibrate the sample between the two-phases. The upper and lower phases were evaporated to dryness and dissolved with methanol to $2 \mathrm{~mL}$ separately. Then they were analyzed by LC. The partition coefficient $(K)$ was expressed as the ratio of the peak area obtained from the upper phase to that of the lower phase.

\section{Preparation of Two-Phase Solvent System and Sample Solution}

Two-phase solvent systems of $n$-hexaneethyl acetate-methanol-water with 
different volume ratio were prepared and the lower phase was used as the mobile phase and upper phase was used as the stationary phase. Each solvent mixture was thoroughly equilibrated in a separation funnel at room temperature after shaking violently. Then the upper phase and lower phase were separated and degassed in a supersonic bath for $30 \mathrm{~min}$ before use. The sample solutions were prepared by dissolving the crude extract in a mixture of upper phase and lower phase in a ratio of $1: 1(v / v)$ of the selected system.

\section{HSCCC Separation Procedure}

The HSCCC system was operated in head to tail mode with the upper phase as stationary phase. The multilayercoiled column was first entirely filled with the upper phase. The lower aqueous mobile phase was then pumped into the head end of the column inlet at a flow-

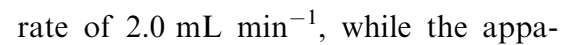
ratus was run at a revolution speed of $800 \mathrm{rpm}$. A crude extract $(600 \mathrm{mg})$ dissolved in $20 \mathrm{~mL}$ mixture of the upper and lower phase was loaded via the injection valve after the system reached hydrodynamic equilibrium. The whole separation experiment was conducted at room temperature $\left(25^{\circ} \mathrm{C}\right)$. The effluent was continuously monitored by a UV detector at $254 \mathrm{~nm}$ and collected with a fraction collector set at $4 \mathrm{~min}$ for each tube. Fractions that contained only ATX $\mathrm{I}$ as identified by LC were combined and evaporated to dryness under vacuum at $40{ }^{\circ} \mathrm{C}$.

\section{LC Analysis and Identification of Target Compound}

The LC analysis was performed with a reversed-phase Phenomenex Luna $\mathrm{C}_{18}$ column $(250 \mathrm{~mm} \times 4.6 \mathrm{~mm}$ I.D., $5 \mu \mathrm{m})$ at room temperature $\left(25^{\circ} \mathrm{C}\right)$. The binary mobile phase consisted of methanol and water in the gradient mode as follows: $0-20 \mathrm{~min}, 30-70 \%$ methanol; 20-25 min, $70-100 \%$ methanol. The

Table 1. The partition coefficient $(K)$ values of Altertoxin I in different biphasic solvent systems used in HSCCC

\begin{tabular}{|ll|}
\hline Solvent system $^{\text {a }}$ & $K$ \\
\hline$n$-Hexane-ethyl acetate-methanol-water $(1: 3: 3: 3, v / v)$ & 0.39 \\
$n$-Hexane-ethyl acetate-methanol-water $(1: 2: 2: 3, v / v)$ & 2.72 \\
$n$-Hexane-ethyl acetate-methanol-water $(2: 5: 5: 6, v / v)$ & 0.91 \\
$n$-Hexane-ethyl acetate-ethanol-water $(2: 5: 5: 6, v / v)$ & 1.27 \\
$n$-Hexane-ethyl acetate-ethanol-water $(3: 5: 6: 6, v / v)$ & 0.32 \\
$n$-Hexane-ethyl acetate-ethanol-water $(4: 5: 5: 6, v / v)$ & 0.62 \\
Dichloromethane-methanol-water $(2: 3: 3, v / v)$ & 0.11 \\
Dichloromethane-methanol-water $(3: 4: 3, v / v)$ & 0.28 \\
\hline
\end{tabular}

a Solvent ratios were by volume

effluent was monitored at $254 \mathrm{~nm}$ and flow rate was at $1.0 \mathrm{~mL} \mathrm{m^{-1 }}$ constantly.

Identification of the HSCCC peak fraction that contained only ATX I was carried out by electrospray ionization mass spectrometry (ESI-MS), ${ }^{1} \mathrm{H}-\mathrm{NMR}$, ${ }^{13} \mathrm{C}-\mathrm{NMR}$ spectra and ${ }^{1} \mathrm{H}-{ }^{13} \mathrm{C}-\mathrm{COSY}$.

\section{Results and Discussion}

\section{Optimization of HSCCC Conditions}

The first and critical step in a HSCCC experiment is the selection of a good solvent system, which can provide an ideal partition coefficient $(K)$ for the target compound. A suitable $K$ value for HSCCC should be preferably in the range of 0.5-1.0. A much smaller $K$ value elutes the solute closer to the solvent front with lower resolution while a much larger $K$ value tends to give better resolution but broader, more dilute peaks due to a longer elution time [25].

In order to achieve efficient resolution of target compound (ATX I), various two phase solvent systems with different ratios were tested. The measured $K$ values for ATX I in these different solvent systems are summarized in Table 1. Among them, two solvent systems, (a) n-hexane-ethyl acetateethanol-water $(4: 5: 5: 6, v / v)$, and (b) $n$-hexane-ethyl acetate-methanol-water (2:5:5:6, v/v) gave good $K$ values at 0.62 and 0.91 , respectively.
For further evaluation of the selected two solvent systems, the ethyl acetate extracts of Alternaria sp. were analyzed by LC and the chromatogram is given in Fig. 1a. Table 2 lists the distributions of the 7 peaks (Fig. 1a) in the two selected solvent systems. In system (a), the $K$ values of peaks 5, $\mathbf{6}$ and $\mathbf{7}$ are so similar that it may result in poor separation. In system (b), the $K$ value of peak $\mathbf{6}$ is significantly different from the other peaks. At room temperature the settling time and the volume ratio (volume of the upper phase divided by that of the lower phase) of the solvent system (b) were $21 \mathrm{~s}$ and 0.38 , respectively. The settling time correlates to the retention of the stationary phase and a good volume ratio could avoid excessive solvent waste [24]. The solvent system (b), providing a reasonable volume ratio between the upper and lower phases with a reasonable range of the settling times in $25 \mathrm{~s}$ was, therefore, used for all later HSCCC runs.

The HSCCC chromatogram is shown in Fig. 2. The peaks $\mathbf{A}-\mathbf{E}$ in Fig. 2 were individually collected and analyzed by LC. Peak $\mathbf{A}$ in the HSCCC chromatogram consisted of peak $\mathbf{1}$ and peak $\mathbf{2}$ in the LC chromatogram (Fig. 1a); Peak B consisted of two peaks (peaks $\mathbf{3}$ and $\mathbf{4}$ ) in the LC chromatogram (Fig. 1a); peak $\mathbf{C}$, peak $\mathbf{D}$ and peak $\mathbf{E}$ were corresponding to peaks 5, 6 and 7 (Fig. 1a), respectively. The peak D eluted from 230 to 260 min contained the target compound ATX I (Fig. 1b) with a purity over $95 \%$. The influence of revolution speed, flow rate of the mobile phase was also investigated. The results indicated that when 

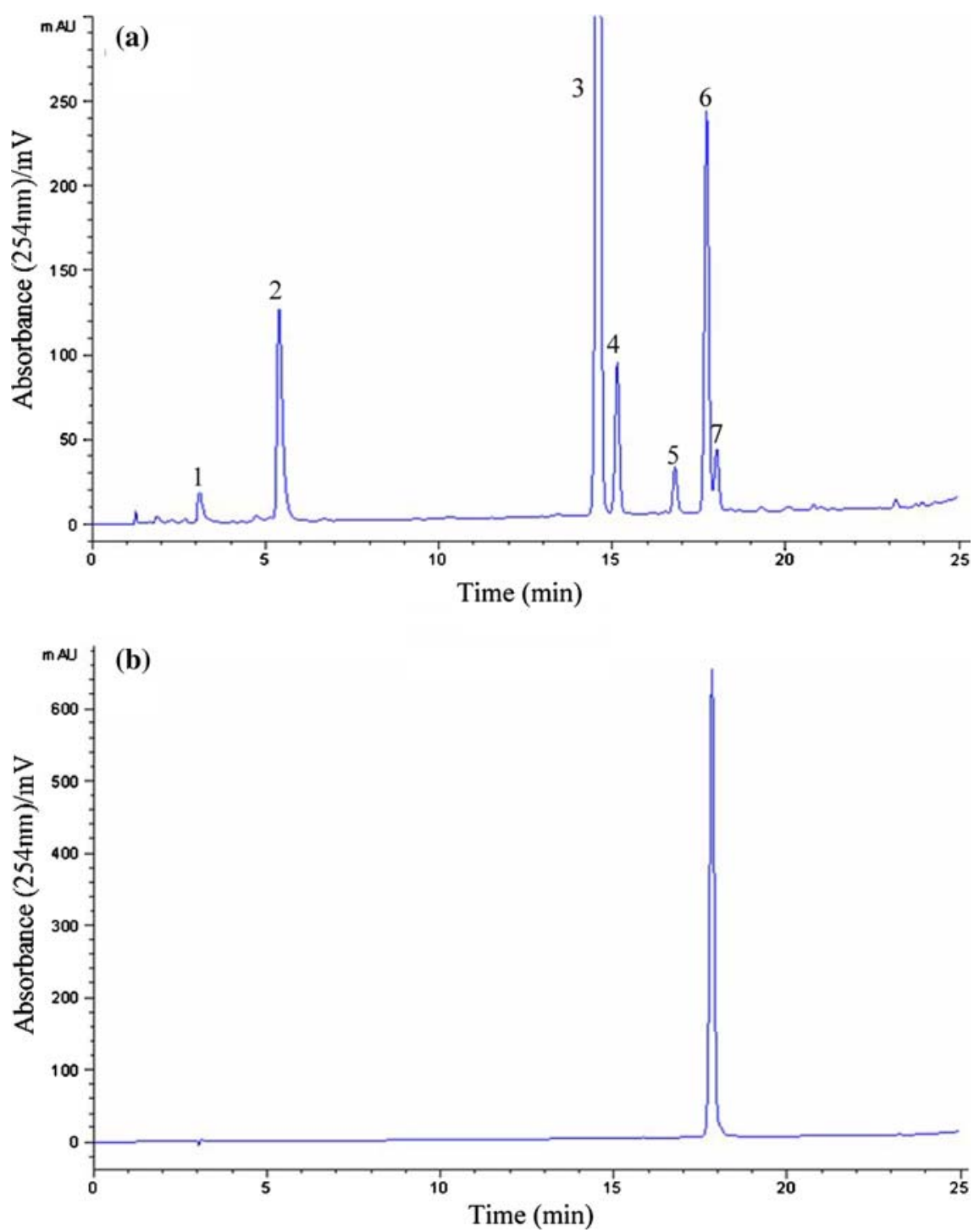

Fig. 1. The results of LC analysis of the crude sample of a Alternaria sp. and $\mathbf{b}$ the purified peak D (ATX I) by HSCCC, column: Phenomenex Luna $C_{18}$ column $(250 \mathrm{~mm} \times 4.6 \mathrm{~mm}$ I.D., $5 \mu \mathrm{m})$ at room temperature, elution: methanol and pure water in gradient mode as follow: 0-20 min, $30-70 \%$ methanol, $20-25 \mathrm{~min}, 70-100 \%$ methanol, flow rate: $1.0 \mathrm{~mL} \mathrm{~min}{ }^{-1}$; detection at $254 \mathrm{~nm}$ (peak $\mathbf{A}$ in the LC chromatogram is peak $\mathbf{1}$ and peak 2; Peak $\mathbf{B}$ in the LC chromatogram is peak $\mathbf{3}$ and peak 4; Peaks $\mathbf{C}, \mathbf{D}$ and $\mathbf{E}$ are peaks 5, $\mathbf{6}$ and 7, respectively)

Table 2. Partition coefficient of the seven peaks in the two solvent systems

\begin{tabular}{|llllllll|}
\hline & Peaks & \multicolumn{1}{l|}{} & & & & & \\
& $\mathbf{1}$ & $\mathbf{2}$ & $\mathbf{3}$ & $\mathbf{4}$ & $\mathbf{5}$ & $\mathbf{6}$ & $\mathbf{7}$ \\
\hline Solvent system (a) & 0.03 & 0.02 & 0.28 & 0.25 & 0.54 & 0.62 & 0.77 \\
Solvent system (b) & 0.02 & 0.02 & 0.22 & 0.23 & 0.58 & 0.91 & 1.21 \\
\hline
\end{tabular}

Solvent systems (a) $n$-hexane-ethyl acetate-ethanol-water (4:5:5:6, v/v), and (b) $n$-hexaneethyl acetate-methanol-water $(2: 5: 5: 6, v / v)$

the flow-rate was $2.0 \mathrm{~mL} \mathrm{~min}^{-1}$, the revolution speed was $800 \mathrm{rpm}$, and separation temperature was $25^{\circ} \mathrm{C}$, retention percentage of the stationary phase was 53\% and good separation results were achieved.

\section{The Yield and Structural Identification}

From $18 \mathrm{~L}$ fermentation culture, a total of $45 \mathrm{mg}$ ATX I with purity of over $95 \%$ were obtained from our HSCCC separation method. The recovery rate of ATX I was $86.8 \%$ based on the original ATX I concentration in the ethyl acetate crude extract analyzed by LC.

The ESI-MS, ${ }^{1} \mathrm{H}-\mathrm{NMR}$ and ${ }^{13} \mathrm{C}-\mathrm{NMR}$ data were used for the structure identification of ATX I, which was in accordance with the literature [28]. ESI-MS: $m / z 353.1[\mathrm{M}+\mathrm{H}]{ }^{+} \cdot{ }^{1} \mathrm{H}-$ NMR (400 MHz, DMSO, TMS) $\delta$ ppm: 8.03 (1 H, d, $J=8.8 \mathrm{~Hz}, \mathrm{H}-12), 7.98$ $(1 \mathrm{H}, \mathrm{d}, J=8.8 \mathrm{~Hz}, \mathrm{H}-1), 7.03(1 \mathrm{H}, \mathrm{d}$, $J=9.2 \mathrm{~Hz}, \mathrm{H}-2), \quad 6.94 \quad(1 \mathrm{H}, \quad \mathrm{d}$, $J=8.8 \mathrm{~Hz}, \mathrm{H}-11), 4.55$ (1 H, m, H-7), 2.85-3.37 (5H, m), $2.30(1 \mathrm{H}, \mathrm{m}, \mathrm{H}-8)$, $2.50(1 \mathrm{H}, \mathrm{m}, \mathrm{H}-5), 12.70(1 \mathrm{H}, 10-\mathrm{OH})$, $12.29(1 \mathrm{H}, 3-\mathrm{OH}), 5.22(1 \mathrm{H}, 7-\mathrm{OH})$, $5.33 \quad(1 \mathrm{H}, \quad 6 \mathrm{a}-\mathrm{OH}) . \quad{ }^{13} \mathrm{C}-\mathrm{NMR}$ (400 MHz, DMSO, TMS) $\delta$ ppm: 206.1 (C-4), 204.2 (C-9), 161.1 (C-10), 160.5 (C-3), 140.8 (C-9b), 138.4 (C-3b), 132.9 (C-12), $132.5 \quad(\mathrm{C}-1), \quad 124.9(\mathrm{C}-12 \mathrm{a})$, 123.6(C-12b) 117.9(C-9a), 116.6 (C-2), 115.6 (C-11), 113.9 (C-3a), 68.1 (C-6a), 64.7 (C-7), 51.5 (C-6b), 47.5 (C-8), 34.9 (C-6), 33.6 (C-5). Two dimension-NMR also supported the structural assignments. The structure of peak $\mathbf{D}$, therefore, was positively identified as ATX I (Fig. 3).

\section{Conclusion}

The HSCCC method has been developed successfully for the purification of ATX I at a semi-preparative scale in this paper. It is a significantly simplified purification procedure to a higher recovery rate as compared with the conventional methods related to column chromatographic techniques, which may cause irreversible adsorption of target compounds to the column. The minimum use of organic solvents with a gentle and fast separation process makes the HSCCC a more environmentally friendly approach. A total amount of $45 \mathrm{mg}$ of ATX I was obtained from the crude sample. The purity of the ATX I was over $95 \%$ after only one-step 


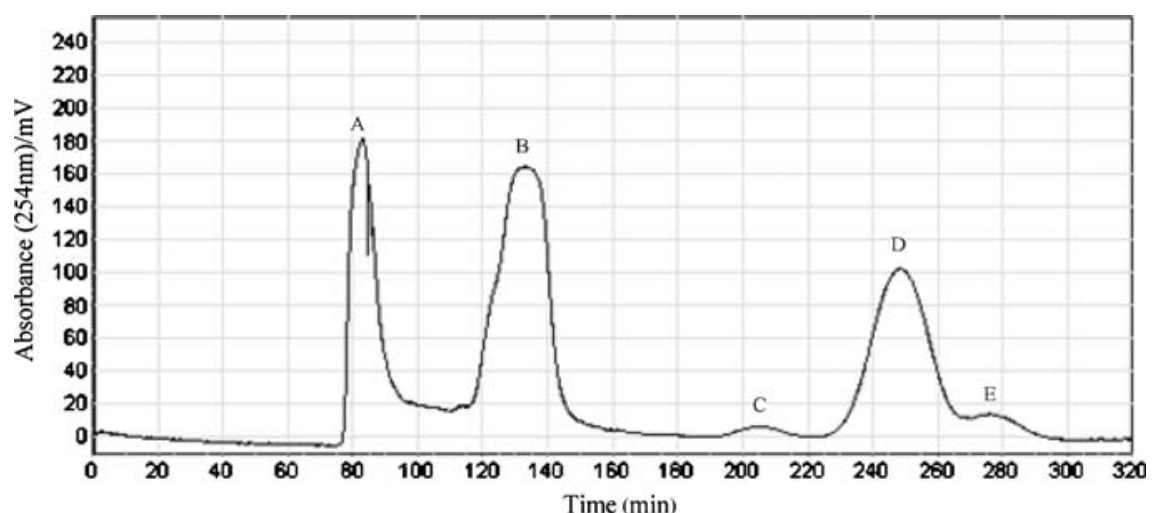

Fig. 2. HSCCC chromatogram of the crude extract of Alternaria sp. in the solvent system of $n$ hexane-ethyl acetate-methanol-water $(2: 5: 5: 6, v / v)$, stationary phase: upper phase, mobile phase: lower phase, flow rate: $2.0 \mathrm{~mL} \mathrm{~min}^{-1}$, revolution speed: $800 \mathrm{rpm}$, temperature: $25^{\circ} \mathrm{C}$, sample size: $600 \mathrm{mg}$ crude extract dissolved in a $20 \mathrm{~mL}$ mixture of upper and lower phase $(1: 1, \mathrm{v} /$ $v$ ) of solvent system, detection at $254 \mathrm{~nm}$ (the fraction of peak $\mathbf{D}$ were collected from 230 to $260 \mathrm{~min}$ and purified to over $95 \%$ estimated by LC analyses)

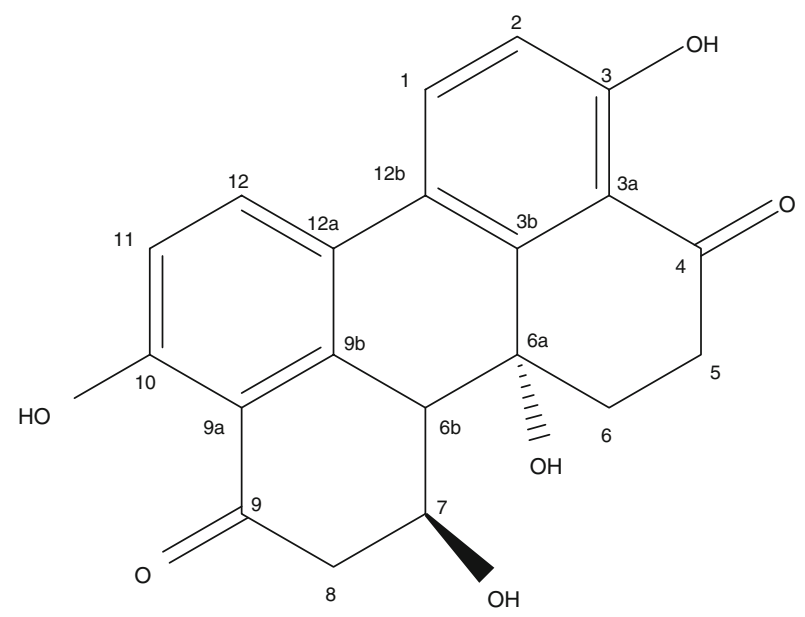

Fig. 3. The structure of Altertoxin I

separation. The ESI-MS and NMR analyses were employed to positively identify the isolated ATX I. The present study indicates that HSCCC is a very powerful technique for the separation and purification of a relatively large amount of highly pure ATX I in a short period.

\section{Acknowledgments}

Financial support from the National Infrastructure of Natural Resources for Science and Technology (Grant no: 2005DKA21203) is gratefully acknowledged.
2. Solfrizzo M, De Girolamo A, Vitti C, Tylkowska K, Grabarkiewicz-Szczesna J, Szopinska D, Dorna H (2005) Food Addit Contam 22:302-308

3. Drusch S, Aumann J (2005) Food Nutr Res 50:33-78

4. Solfrizzo M, De Girolamo A, Vitti C, Visconti A, Van Den Bulk R (2004) J AOAC Int 87:101-106

5. Nielsen KF (2003) Fungal Genet Biol 39:103-117

6. Schrader TJ, Cherry W, Soper K, Langlois I, Vijay HM (2001) Teratog Carcinog Mutagen 21:261-274

7. Woody MA, Chu FS (1992) In: Chelkowski J, Visconti A (eds) Alternaria: biology, plant diseases and metabolites, Elsevier, New York, pp 409-434

8. Panigrahi S (1997) In: Miller JD, Trenholm, HL (eds) Handbook of plant and fungal toxicants. CRC Press, Boca Raton, pp 319-337

9. Schrader TJ, Cherry W, Soper K, Langlois I (2006) Mutat Res 606:61-71

10. Liu GT, Qian YZ; Zhang P, Dong ZM, Shi ZY, Zhen YZ, Miao J, Xu YM (1991) In: O'Neill IK, Chen J, Bartsch J (eds) Relevance to human cancer of N-nitroso compounds, tobacco and mycotoxins, Lyon: International Agency for Research on Cancer, pp 258-262

11. Ikins WG (1991) In: Fung DYC, Matthews RF (eds) Instrumental methods of quality assurance in foods, Marcel Dekker, New York, pp 117-154

12. Li FQ, Yoshizawa T (2000) J Agric Food Chem 48:2920-2924

13. Hasan HAH (1996) Acta Microbiol Immunol Hung 43:125-133

14. Li FQ, Toyazaki N, Yoshizawa T (2001) J Food Prot 64:567-571

15. Scott PM, Weber D, Kanhere SR (1997) J Chromatogr A 765:255-263

16. Nawaz S, Scudamore KA, Rainbird SC (1997) Food Addit Contam 14:249-262

17. Stack ME, Prival MJ (1986) Appl Environ Microbiol 52:718-722

18. Osborne LC, Jones VI, Peeler JT, Larkin EP (1988) Toxicol In Vitro 2:97-102

19. Boutin BK, Peeler JT, Twedt RM (1989) J Toxicol Environ Health 26:75-81

20. Lehmann L, Wagner J, Metzler M (2006) Food Chem Toxicol 44:398-408

21. Stinson EE, Osman SF, Pfeffer PE (1982) J Org Chem 47:4110-4113

This article is distributed under the term of the Creative Commons Attribution Noncommercial License which permits any noncommercial use, distribution, and reproduction in any medium, provided the original author(s) and source are credited.

\section{References}

1. Bottalico A, Logrieco A (1998) In: Sinha HK, Bhatnagar D (eds) Mycotoxins in agriculture and food safety. Marcel Dekker, New York, pp 65-108
22. Chu F (1981) J Am Oil Chem Soc 58:A1006-A1008

23. Ito Y (1986) CRC Crit Rev Anal Chem $17: 65-143$

24. Oka H, Harada KI, Ito Y (1998) J Chromatogr A 812:35-52

25. Ito Y. (2005) J Chromatogr A 1065:145168

26. He J, Yang R, Zhou T, Tsao R, Young JC, Zhu H, Li XZ, Boland GJ (2007) J Chromatogr A 1151:187-192

27. Friesen JB, Pauli GF (2007) J Chromatogr A 1151:51-59

28. Okuno T, Natsume I, Sawai K, Sawamura K (1983) Tetrahedron Lett 24:56535656 